\title{
Resolution of Non-Small-Cell Lung Cancer after Withdrawal of Anti-TNF Therapy
}

TO THE EDITOR: We report the development of locally advanced non-small-cell lung cancer (tumor-node-metastasis [TNM] stage T4N2M0) in a 69-year-old woman with Crohn's colitis. Her condition had been diagnosed in 2000 and had required treatment with methotrexate (from 2003 to the present), together with anti-tumor necrosis factor (TNF) therapies - initially, episodic infliximab (from 2000 to 2004) and thereafter, maintenance adalimumab, until the tumor was diagnosed in June 2006. Remarkably, the tumor expressed TNF receptors and underwent regression and sustained remission on withdrawal of anti-TNF therapy, with no other therapeutic intervention.

In 1999, the patient, a former smoker (35 packyears), was noted to have finger clubbing. A computed tomographic (CT) scan showed interstitial lung disease; she had normal pulmonary-function tests but remained under respiratory review. On repeat CT in June 2006, a pleural-based spiculated mass, 2.5 by $2.5 \mathrm{~cm}$, was noted in the right lower lobe (Fig. 1A) with two small satellite appendages. Mediastinal and hilar lymphadenopathy was present. CT-guided biopsy of the main lesion confirmed non-small-cell lung cancer, which was positive for TNF receptors type 1 and type 2 on immunostaining (data not shown). Adalimumab was withdrawn, but methotrexate was continued. The patient did not undergo surgery and did not receive radiotherapy, chemotherapy, or biologic therapy. In April 2007, on repeat CT scanning, there was virtually no evidence of primary lung tumor, nodules, or lymphadenopathy (Fig. 1B). At the time of this report, she was in complete clinical and radiologic remission.

There is now real concern about the risk of lymphoma in patients exposed to anti-TNF therapies. ${ }^{1,2}$ In addition, there are reports of lung cancer, all in heavy smokers given infliximab. In the Mayo Clinic series of 500 patients with Crohn's disease, two lung cancers, deemed "possibly related" to infliximab, were reported in elderly smokers. ${ }^{3}$ In a 24-week trial involving patients with chronic obstructive pulmonary disease, a total of 12 cancers, including 6 lung cancers, were diagnosed in 157 patients treated with infliximab. ${ }^{4}$ In our unit, we have seen three lung cancers in patients treated with anti-TNF therapies,
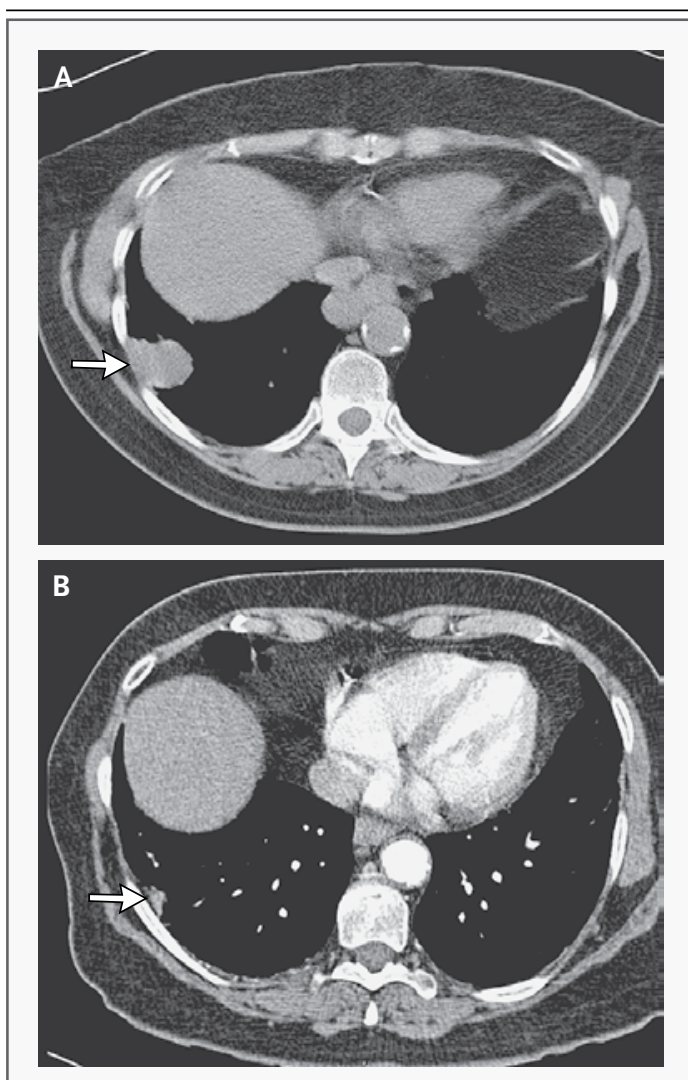

Figure 1. Representative CT Scans Obtained during and after the Withdrawal of Anti-Tumor Necrosis Factor (TNF) Therapy.

A CT scan obtained in June 2006, when the patient was receiving anti-TNF therapy, shows the primary nonsmall-cell lung tumor in the right lower lobe (Panel A, arrow). Mediastinal lymph-node enlargement was seen on CT studies and was confirmed as necrotic tumor within malignant lymph nodes on endoscopic ultrasound-guided biopsy. CT scans obtained after the withdrawal of adalimumab and in the absence of any specific antitumor therapy, in April 2007 (not shown) and July 2007 (Panel B), showed minimal disease at the site of the primary lung tumor (arrow in Panel B), and the lymphadenopathy had resolved.

all of whom were smokers or former smokers over the age of 65 years.

The remarkable aspect of our case is the sustained remission on withdrawal of anti-TNF therapy. The overexpression of TNF receptors type 1 and type 2 implicates TNF blockade in carcinogenesis. This hypothesis is supported by data demon- 
strating a critical role of adaptive immunity in maintaining tumor dormancy after exposure to a carcinogen in cigarette smoke. ${ }^{5}$ We recommend special vigilance in the use of anti-TNF therapy in patients with a history of smoking or chronic lung disease, especially those over the age of 65 years.

\section{Charles W. Lees, M.R.C.P.}

Janet Ironside, F.R.C.P.

Western General Hospital

Edinburgh EH4 2XU, United Kingdom

William A.H. Wallace, F.R.C.P.Ed., F.R.C.Path.

Royal Infirmary of Edinburgh

Edinburgh EH16 4TJ, United Kingdom

Jack Satsangi, D.Phil.

Western General Hospital

Edinburgh EH4 2XU, United Kingdom

j.satsangi@ed.ac.uk

Dr. Lees reports receiving lecture fees from Schering-Plough and Abbott; and Dr. Satsangi, consulting and lecture fees from
Abbott and UCB and consulting fees and grant support from Schering-Plough. No other potential conflict of interest relevant to this letter was reported.

1. Bongartz T, Sutton AJ, Sweeting MJ, Buchan I, Matteson EL, Montori V. Anti-TNF antibody therapy in rheumatoid arthritis and the risk of serious infections and malignancies: systematic review and meta-analysis of rare harmful effects in randomized controlled trials. JAMA 2006;295:2275-85. [Erratum, JAMA 2006;295:2482.]

2. Mackey AC, Green L, Liang LC, Dinndorf P, Avigan M. Hepatosplenic $\mathrm{T}$ cell lymphoma associated with infliximab use in young patients treated for inflammatory bowel disease. J Pediatr Gastroenterol Nutr 2007;44:265-7.

3. Colombel JF, Loftus EV Jr, Tremaine WJ, et al. The safety profile of infliximab in patients with Crohn's disease: the Mayo Clinic experience in 500 patients. Gastroenterology 2004;126: 19-31.

4. Rennard SI, Fogarty C, Kelsen S, et al. The safety and efficacy of infliximab in moderate to severe chronic obstructive pulmonary disease. Am J Respir Crit Care Med 2007;175:926-34.

5. Koebel CM, Vermi W, Swann JB, et al. Adaptive immunity maintains occult cancer in an equilibrium state. Nature 2007; 450:903-7.

Correspondence Copyright @ 2008 Massachusetts Medical Society.

Letters to the Editor are considered for publication, subject to editing and abridgment, provided they do not contain material that has been submitted or published elsewhere. Please note the following: $\cdot$ Letters in reference to a Journal article must not exceed 175 words (excluding references) and must be received within 3 weeks after publication of the article. Letters not related to a Journal article must not exceed 400 words. All letters must be submitted over the Internet at http://authors.nejm.org. -A letter can have no more than five references and one figure or table. • A letter can be signed by no more than three authors. - Financial associations or other possible conflicts of interest must be disclosed. (Such disclosures will be published with the letters. For authors of Journal articles who are responding to letters, this information appears in the published articles.) -Include your full mailing address, telephone number, fax number, and e-mail address with your letter.

Our Web site: http://authors.nejm.org

We cannot acknowledge receipt of your letter, but we will notify you when we have made a decision about publication. Letters that do not adhere to these instructions will not be considered. Rejected letters and figures will not be returned. We are unable to provide prepublication proofs. Submission of a letter constitutes permission for the Massachusetts Medical Society, its licensees, and its assignees to use it in the Journal's various print and electronic publications and in collections, revisions, and any other form or medium. 